\title{
On the solutions of the nonlinear Liouville hierarchy 1
}

\author{
V. O. SHTYK \\ Institute of Mathematics of NAS of Ukraine, 3 Tereshchenkivs'ka Str., 01601 Kyiv-4, Ukraine \\ E-mail: shtyk@imath.kiev.ua
}

\begin{abstract}
.
We investigate the initial-value problem of the non-linear Liouville hierarchy. For the general form of the interaction potential we construct an explicit solution in terms of an expansion over particle clusters whose evolution is described by the corresponding-order cumulant of evolution operators of a system of finitely many particles. For the initial data from the space of integrable functions the existence of a strong solution of the Cauchy problem is proved.
\end{abstract}

Key words: nonlinear Liouville hierarchy; cumulant; cluster.

2000 Mathematics Subject Classification: 82C05; 37A60.

\section{Introduction}

The nonlinear Liouville hierarchy that describes the evolution of correlation functions, arises in many problems of statistical mechanics concerning many-particle systems [1, 2, 7]. However, today it is still insufficiently studied from the mathematical point of view.

It is well known [4], that all possible states of a classical system of a finite number of particles are described by the functions interpreted as probability density functions. These functions are solutions of the initial-value problem of the Liouville hierarchy - the first-order partial differential equations, whose characteristic equations are Hamilton equations. If the state of a system is presented in terms of a cluster expansion in new (correlation) functions one evidently obtains an equivalent description of this state. Now evolution of the correlation functions is determined by the nonlinear Liouville hierarchy - certain nonlinear first-order partial differential equations.

In this paper an explicit solution of such nonlinear equations is constructed and presented as an expansion in terms of particle clusters whose evolution is described by a cumulant (semi-invariant) of the evolution operators. The latter are determined by the solutions of the characteristic equations of the linear Liouville equation, i.e., the Hamilton equations. The interaction potential of the general form is considered, which makes it possible to describe the general structure of the generator of the nonlinear Liouville hierarchy. The existence of a strong solution of the Cauchy problem with initial data from the space of integrable functions is proved. A formal treatment of the nonlinear Liouville hierarchy for the case of a pairwise interaction potential, was given by Bogolyubov and Green [1, 2].

It should be noted that the nonlinear Liouville hierarchy is basic in the substantiation of the derivation of the nonlinear Bogolyubov hierarchy [2] whose solutions describe the correlation dynamics of infinite systems of particles. Moreover, this concerns the mathematical substantiation of the correlation-weakening principle. The correlation functions may be employed to directly calculate the specific characteristics of the system, i.e., fluctuations, defined as the average values of the square deviations of an observable from its average value, as well as macroscopic values which are not averages of observables. The construction of the nonlinear Bogolyubov hierarchy and the analysis of the solutions thereof for on the basis of the results obtained here will be given in an other paper.

\footnotetext{
${ }^{1}$ This work was partially supported by the WTZ grant
} 


\section{Initial-Value Problem of Nonlinear Liouville Hierarchy}

Let us consider a system of non-fixed number of identical particles with phase coordinates $x_{i}=$ $\left(q_{i}, p_{i}\right) \in \mathbb{R}^{\nu} \times \mathbb{R}^{\nu}, \nu \geq 1$. The relevant Hamiltonian is given by the formula

$$
H_{n}=\sum_{i=1}^{n} \frac{p_{i}^{2}}{2}+\sum_{k=1}^{n} \sum_{i_{1}<i_{2}<\ldots<i_{k}=1}^{n} \Phi_{k}\left(q_{i_{1}}, q_{i_{2}}, \ldots, q_{i_{k}}\right),
$$

where $\Phi_{k}$ is the $k$-th-order interaction potential. In what follows we assume that the interaction potential $\Phi_{k}, k \geq 1$, satisfies the necessary conditions which provide the existence of global in time solutions of the Hamilton equations.

The state of the system can be described by a sequence $g(t)=\left(0, g_{1}\left(t, x_{1}\right), \ldots\right.$ $\left.\ldots, g_{n}\left(t, x_{1}, \ldots, x_{n}\right), \ldots\right)$, of correlation functions $g_{n}\left(t, x_{1}, \ldots, x_{n}\right)$ defined on the phase space $\mathbb{R}^{\nu n} \times \mathbb{R}^{\nu n}, \nu \geq 1$ and symmetric with respect to the permutations of arguments $x_{1}, \ldots, x_{n}$.

The evolution of the states of the above system is described by the initial-value problem of the nonlinear Liouville hierarchy

$$
\begin{aligned}
& \frac{d}{d t} g_{n}(t, Y)=\left(-\mathcal{L}_{|Y|}(Y)\right) g_{n}(t, Y)+
\end{aligned}
$$

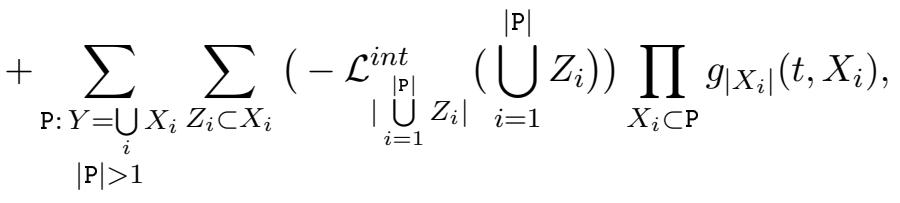

$$
\begin{aligned}
& \left.g_{n}(t, Y)\right|_{t=0}=g_{n}(0, Y), \quad n \geq 1,
\end{aligned}
$$

where the following notation is used $Y \equiv\left(x_{1}, \ldots, x_{n}\right),|Y|=n$ denotes the number of elements of the set $Y, \sum_{\mathrm{P}: Y=\bigcup_{i} X_{i}}$ is the sum over all possible (in this case) decompositions $\mathrm{P}$ of the set $Y$ into $|\mathrm{P}|$ nonempty mutually disjoint subsets, $\sum_{Z_{i} \subset X_{i}}$ - is the sum over all nonempty subsets $Z_{i} \subset X_{i}$. The Liouville operator $\mathcal{L}_{n}$ for the Hamiltonian $H_{n}$ is described by the formulas

$$
\begin{aligned}
& \mathcal{L}_{n} \equiv \mathcal{L}_{n}\left(x_{1}, \ldots, x_{n}\right)=\sum_{i=1}^{n}\left\langle p_{i}, \frac{\partial}{\partial q_{j}}\right\rangle+\sum_{k=2}^{n} \sum_{i_{1}<i_{2}<\ldots<i_{k}=1}^{n} \mathcal{L}_{k}^{\text {int }}\left(x_{i_{1}}, x_{i_{2}}, \ldots, x_{i_{k}}\right), \\
& \mathcal{L}_{k}^{\text {int }}\left(x_{i_{1}}, x_{i_{2}}, \ldots, x_{i_{k}}\right)=-\sum_{j=1}^{n}\left\langle\frac{\partial}{\partial q_{j}} \Phi_{k}\left(q_{i_{1}}, q_{i_{2}}, \ldots, q_{i_{k}}\right), \frac{\partial}{\partial p_{j}}\right\rangle,
\end{aligned}
$$

where $\langle\cdot, \cdot\rangle$ - is the scalar product.

The simplest examples of the nonlinear Liouville hierarchy are given by:

$$
\begin{aligned}
& \frac{d}{d t} g_{1}\left(t, x_{1}\right)=-\mathcal{L}_{1}\left(x_{1}\right) g_{1}\left(t, x_{1}\right) \\
& \frac{d}{d t} g_{2}\left(t, x_{1}, x_{2}\right)=-\mathcal{L}_{2}\left(x_{1}, x_{2}\right) g_{2}\left(t, x_{1}, x_{2}\right)-\mathcal{L}_{2}^{i n t}\left(x_{1}, x_{2}\right) g_{1}\left(t, x_{1}\right) g_{1}\left(t, x_{2}\right) \\
& \frac{d}{d t} g_{3}\left(t, x_{1}, x_{2}, x_{3}\right)=-\mathcal{L}_{3}\left(x_{1}, x_{2}, x_{3}\right) g_{3}\left(t, x_{1}, x_{2}, x_{3}\right)+ \\
& \quad+\left(-\mathcal{L}_{2}^{i n t}\left(x_{1}, x_{2}\right)-\mathcal{L}_{2}^{\text {int }}\left(x_{1}, x_{3}\right)-\mathcal{L}_{3}^{i n t}\left(x_{1}, x_{2}, x_{3}\right)\right) g_{1}\left(t, x_{1}\right) g_{2}\left(t, x_{2}, x_{3}\right)+ \\
& \quad+\left(-\mathcal{L}_{2}^{\text {int }}\left(x_{1}, x_{2}\right)-\mathcal{L}_{2}^{\text {int }}\left(x_{2}, x_{3}\right)-\mathcal{L}_{3}^{\text {int }}\left(x_{1}, x_{2}, x_{3}\right)\right) g_{1}\left(t, x_{2}\right) g_{2}\left(t, x_{1}, x_{3}\right)+ \\
& \quad+\left(-\mathcal{L}_{2}^{i n t}\left(x_{1}, x_{3}\right)-\mathcal{L}_{2}^{\text {int }}\left(x_{2}, x_{3}\right)-\mathcal{L}_{3}^{\text {int }}\left(x_{1}, x_{2}, x_{3}\right)\right) g_{1}\left(t, x_{3}\right) g_{2}\left(t, x_{1}, x_{2}\right)+ \\
& \quad-\mathcal{L}_{3}^{\text {int }}\left(x_{1}, x_{2}, x_{3}\right) g_{1}\left(t, x_{1}\right) g_{1}\left(t, x_{2}\right) g_{1}\left(t, x_{3}\right)
\end{aligned}
$$


We note that in the case of a pairwise interaction potential, $(k=2)$, the nonlinear Liouville hierarchy (11) is simpler. For example, expression for $g_{3}(t)$ does not contain members with $\mathcal{L}_{3}^{\text {int }}$. This case was considered by Green [2].

In this work the solution (1)-(2) is shown to be given by the formula

$$
g_{n}(t, Y)=\sum_{\mathrm{P}: Y=\bigcup_{i} X_{i}} \mathfrak{A}_{|\mathrm{P}|}\left(t, Y_{X_{i}}\right) \prod_{X_{i} \subset \mathrm{P}} g_{\left|X_{i}\right|}\left(0, X_{i}\right)
$$

where $Y=\left(x_{1}, \ldots, x_{n}\right), Y_{X_{i}} \equiv\left(X_{1}, \ldots, X_{|\mathrm{P}|}\right), \sum_{\mathrm{P}: Y=\bigcup_{i} X_{i}}-$ the sum of all possible (in this case) decomposition $\mathrm{P}$ of the set $Y$ into $|\mathrm{P}|$ nonempty mutually disjoint subsets $X_{i}$. The evolution operator $\mathfrak{A}_{|\mathrm{P}|}(t)$ i.e., the cumulant (semi-invariant) of the order $|\mathrm{P}|$ is given by the expression [3, 5 ]

$$
\mathfrak{A}_{|\mathrm{P}|}\left(t, Y_{X_{i}}\right)=\sum_{\mathrm{P}^{\prime}: Y_{X_{i}}=\bigcup \begin{array}{l}
Z_{k} \\
Z_{k}
\end{array}}(-1)^{\left|\mathrm{P}^{\prime}\right|-1}\left(\left|\mathrm{P}^{\prime}\right|-1\right) ! \prod_{Z_{k} \subset \mathrm{P}^{\prime}} S_{\left|Z_{k}\right|}\left(-t, Z_{k}\right) .
$$

The evolution operators $S_{n}(-t), n \geq 1$, are given by

$$
\left(S_{n}(-t) g_{n}(0)\right)\left(x_{1}, \ldots, x_{n}\right)=g_{n}\left(0, X_{1}\left(-t, x_{1}, \ldots, x_{n}\right), \ldots, X_{n}\left(-t, x_{1}, \ldots, x_{n}\right)\right),
$$

where $\left\{X_{i}\left(-t, x_{1}, \ldots, x_{n}\right)\right\}_{i=1}^{n}$ is the solution of the relevant initial-value problem for the Hamilton equations. The properties of a group of evolution operator (6) are described in [6].

Let us consider the simplest examples of expansions (44) with the following notation: the argument $x_{i} \cup x_{j}$ implies, that two particles $i$-th and $j$-th evolve as a cluster. Thus, if the arguments of the operator are clusters, they enter on equal terms the expansions in series of the evolution operators $S_{n}(-t)$, the order of the cumulant being equal to the number of its cluster arguments .

$$
\begin{aligned}
& g_{1}\left(t, x_{1}\right)=\mathfrak{A}_{1}\left(t, x_{1}\right) g_{1}\left(0, x_{1}\right), \\
& g_{2}\left(t, x_{1}, x_{2}\right)=\mathfrak{A}_{1}\left(t, x_{1} \cup x_{2}\right) g_{2}\left(0, x_{1}, x_{2}\right)+\mathfrak{A}_{2}\left(t, x_{1}, x_{2}\right) g_{1}\left(0, x_{1}\right) g_{1}\left(0, x_{2}\right), \\
& g_{3}\left(t, x_{1}, x_{2}, x_{3}\right)=\mathfrak{A}_{1}\left(t, x_{1} \cup x_{2} \cup x_{3}\right) g_{3}\left(0, x_{1}, x_{2}, x_{3}\right)+\mathfrak{A}_{2}\left(t, x_{1}, x_{2} \cup x_{3}\right) g_{1}\left(0, x_{1}\right) g_{2}\left(0, x_{2}, x_{3}\right)+ \\
& \quad+\mathfrak{A}_{2}\left(t, x_{1} \cup x_{3}, x_{2}\right) g_{1}\left(0, x_{2}\right) g_{2}\left(0, x_{1}, x_{3}\right)+\mathfrak{A}_{2}\left(t, x_{1} \cup x_{2}, x_{3}\right) g_{1}\left(0, x_{3}\right) g_{2}\left(0, x_{1}, x_{2}\right)+ \\
& \quad+\mathfrak{A}_{3}\left(t, x_{1}, x_{2}, x_{3}\right) g_{1}\left(0, x_{1}\right) g_{1}\left(0, x_{2}\right) g_{1}\left(0, x_{3}\right),
\end{aligned}
$$

where, for example, the cumulants (5) are given by

$$
\begin{aligned}
& \mathfrak{A}_{2}\left(t, x_{1} \cup x_{2}, x_{3}\right)=S_{3}\left(-t, x_{1}, x_{2}, x_{3}\right)-S_{1}\left(-t, x_{3}\right) S_{2}\left(-t, x_{1}, x_{2}\right) \\
& \mathfrak{A}_{3}\left(t, x_{1}, x_{2}, x_{3}\right)=S_{3}\left(-t, x_{1}, x_{2}, x_{3}\right)- \\
& \quad-S_{1}\left(-t, x_{1}\right) S_{2}\left(-t, x_{2}, x_{3}\right)-S_{1}\left(-t, x_{2}\right) S_{2}\left(-t, x_{1}, x_{3}\right)-S_{1}\left(-t, x_{3}\right) S_{2}\left(-t, x_{1}, x_{2}\right)+ \\
& \quad+2 ! S_{1}\left(-t, x_{1}\right) S_{1}\left(-t, x_{2}\right) S_{1}\left(-t, x_{3}\right) .
\end{aligned}
$$

Formally, the nonlinear Liouville hierarchy (II) can be derived from the sequence of (linear) Liouville equations which describe the evolution of all possible states of the system of non-fixed number of particles (the sequence $D(t)=\left(1, D_{1}\left(x_{1}\right), \ldots, D_{n}\left(x_{1}, \ldots, x_{n}\right), \ldots\right)$, with the function $D_{n}(t)$ being regarded as the density of probability distribution of the $n$-particles system) [4]

$$
\begin{aligned}
& \frac{\partial D_{n}(t)}{\partial t}=-\mathcal{L}_{n} D_{n}(t), \\
& \left.D_{n}(t)\right|_{t=0}=D_{n}(0), \quad n \geq 1,
\end{aligned}
$$


provided the state of the system is described in terms of correlation functions, i.e.,

$$
g_{n}(t, Y)=\sum_{\mathrm{P}: Y=\bigcup_{i} X_{i}}(-1)^{|\mathrm{P}|-1}(|\mathrm{P}|-1) ! \prod_{X_{i} \subset \mathrm{P}} D_{\left|X_{i}\right|}\left(t, X_{i}\right),
$$

or at the initial time instant

$$
g_{n}(0, Y)=\sum_{\mathrm{P}: Y=\bigcup_{i} X_{i}}(-1)^{|\mathrm{P}|-1}(|\mathrm{P}|-1) ! \prod_{X_{i} \subset \mathrm{P}} D_{\left|X_{i}\right|}\left(0, X_{i}\right), \quad n \geq 1,
$$

where $Y \equiv\left(x_{1}, \ldots, x_{n}\right), \sum_{\mathrm{P}}$ is the sum over all decompositions $\mathrm{P}$ of the set $Y$ into $|\mathrm{P}|$ nonempty mutually disjoint subsets $X_{i}$.

For example,

$$
\begin{aligned}
& g_{1}\left(t, x_{1}\right)=D_{1}\left(t, x_{1}\right), \\
& g_{2}\left(t, x_{1}, x_{2}\right)=D_{2}\left(t, x_{1}, x_{2}\right)-D_{1}\left(t, x_{1}\right) D_{1}\left(t, x_{2}\right) .
\end{aligned}
$$

The solution (4) of the initial-value problem (11)-(2) can be formally derived from(7) and (8) provided one takes into account that, within the context of (8), we have

$$
D_{\left|X_{i}\right|}\left(0, X_{i}\right)=\sum_{\mathrm{P}_{2}: X_{i}=\bigcup_{k}} \prod_{Z_{k}} g_{Z_{k} \subset \mathrm{P}_{2}}\left(0, Z_{k}\right) .
$$

Then, inasmuch as the solution of the Liouville equation is given by

$$
D_{n}(t)=S_{n}(-t) D_{n}(0)
$$

where $S_{n}(-t)$ is determined by the solutions of Hamiltonian equations according to (6), we have

$$
\begin{aligned}
g_{n}(t, Y)=\sum_{\mathrm{P}_{1}: Y=\bigcup_{i} X_{i}}(-1)^{\left|\mathrm{P}_{1}\right|-1}\left(\left|\mathrm{P}_{1}\right|-1\right) ! \prod_{X_{i} \subset \mathrm{P}_{1}} \times \\
\quad \times S_{\left|X_{i}\right|}\left(-t, X_{i}\right) \sum_{\mathrm{P}_{2}: X_{i}=\bigcup_{k}} \prod_{Z_{k}} \prod_{Z_{k} \subset \mathrm{P}_{2}} g_{\left|Z_{k}\right|}\left(0, Z_{k}\right) .
\end{aligned}
$$

Having collected in (9) the terms with similar product of functions $g_{\left|Z_{k}\right|}\left(0, Z_{k}\right)$, one obtains (4).

\section{The existence theorem for the initial-value problem of the nonlinear Liouville hierarchy.}

Suppose $L_{n}^{1}$ is the Banach space of integrable functions $g_{n}\left(x_{1}, \ldots, x_{n}\right)$, defined on the phase space $\mathbb{R}^{\nu n} \times \mathbb{R}^{\nu n}, \nu \geq 1$ of $n$-particle system, symmetric under the perturbations of arguments. The norm of an element $g_{n}$ of $L_{n}^{1}$ is denoted by

$$
\left\|g_{n}\right\|=\int_{\mathbb{R}^{\nu} \times \mathbb{R}^{\nu}} d x_{1} \ldots d x_{n}\left|g_{n}\left(x_{1}, \ldots, x_{n}\right)\right|
$$

$L_{n, 0}^{1} \subset L_{n}^{1}$ is a subspace of continuously differentiable functions with compact supports.

The following theorem is true. 
Theorem 1. If $g_{n}(0) \in L_{n, 0}^{1} \subset L_{n}^{1}, n \geq 1$, then for $t \in \mathbb{R}^{1}$ there exists a unique strong solution to the initial-value problem (11)-(圆) of the nonlinear Liouville hierarchy (11) given by

$$
g_{n}(t, Y)=\sum_{P: Y=\bigcup_{i} X_{i}} \mathfrak{A}_{|P|}\left(t, Y_{X_{i}}\right) \prod_{X_{i} \subset P} g_{\left|X_{i}\right|}\left(0, X_{i}\right),
$$

where $\mathfrak{A}_{|P|}(t)$ is a cumulant (semi-invariant) of the order $|P|$ (5) of the evolution operators (6).

Доказательство. Let us show that the expansion (4) is defined in $L_{n}^{1}$.

Indeed, within the context of the corollary of the Liouville theorem [6] (isometric property of operators $\left.S_{\left|Z_{k}\right|}\left(-t, Z_{k}\right)\right)$ the following estimate is valid

$$
\begin{aligned}
& \left\|g_{n}(t)\right\|=\int d Y\left|\sum_{\mathrm{P}: Y=\bigcup_{i} X_{i}} \mathfrak{A}_{|\mathrm{P}|}\left(t, Y_{X_{i}}\right) \prod_{X_{i} \subset \mathrm{P}} g_{\left|X_{i}\right|}\left(0, X_{i}\right)\right|= \\
& =\int d Y\left|\sum_{\mathrm{P}: Y=\bigcup_{i} X_{i} \mathrm{P}^{\prime}: Y_{X_{i}}=\bigcup \begin{array}{l}
k \\
Z_{k}
\end{array}}(-1)^{\left|\mathrm{P}^{\prime}\right|-1}\left(\left|\mathrm{P}^{\prime}\right|-1\right) ! \prod_{Z_{k} \subset \mathrm{P}^{\prime}} S_{\left|Z_{k}\right|}\left(-t, Z_{k}\right) \prod_{X_{i} \subset \mathrm{P}} g_{\left|X_{i}\right|}\left(0, X_{i}\right)\right| \leq \\
& \leq \sum_{\mathrm{P}: Y=\bigcup_{i} X_{i} \mathrm{P}^{\prime}: Y_{X_{i}}=\bigcup_{k} Z_{k}}\left(\left|\mathrm{P}^{\prime}\right|-1\right) ! \prod_{X_{i} \subset \mathrm{P}}\left\|g_{\left|X_{i}\right|}(0)\right\| \leq n ! e^{n+1} \sum_{\mathrm{P}: Y=\bigcup_{i} X_{i}} \prod_{X_{i} \subset \mathrm{P}}\left\|g_{\left|X_{i}\right|}(0)\right\|<\infty,
\end{aligned}
$$

i.e., $g_{n}(t) \in L_{n}^{1}$ for any $t \in \mathbb{R}^{1}$.

Let us prove that the expansion (4) is a strong solution of the Cauchy problem of the nonlinear Liouville hierarchy (1) -(2).

To do this we first differentiate the functions $g_{n}(t)$ with respect to time with regard for the point-by-point convergence. Let $g_{n}(0) \in L_{n, 0}^{1}$, then inasmuch

$$
\frac{d}{d t} S_{\left|X_{i}\right|}\left(-t, X_{i}\right)=-\mathcal{L}_{\left|X_{i}\right|}\left(X_{i}\right) S_{\left|X_{i}\right|}\left(-t, X_{i}\right),
$$

where the Liouvilian $\mathcal{L}_{\left|X_{i}\right|}$ is defined by (3), and according to (92), for each fixed point $Y$ on any compact from $\mathbb{R}^{\nu n} \times \mathbb{R}^{\nu n}$, we have

$$
\begin{aligned}
& \frac{d}{d t} g_{n}(t, Y)=\frac{d}{d t} \sum_{\mathrm{P}: Y=\bigcup_{i} X_{i}} \mathfrak{A}_{|\mathrm{P}|}\left(t, Y_{X_{i}}\right) \prod_{X_{i} \subset \mathrm{P}_{1}} g_{\left|X_{i}\right|}\left(0, X_{i}\right)= \\
& =\frac{d}{d t} \sum_{\mathrm{P}_{1}: Y=\bigcup_{i} X_{i}}(-1)^{\left|\mathrm{P}_{1}\right|-1}\left(\left|\mathrm{P}_{1}\right|-1\right) ! \prod_{X_{i} \subset \mathrm{P}_{1}} S_{\left|X_{i}\right|}\left(-t, X_{i}\right) \sum_{\mathrm{P}_{2}: X_{i}=\bigcup_{k}} \prod_{Z_{k}} g_{Z_{k} \subset \mathrm{P}_{2}} g_{\left|Z_{k}\right|}\left(0, Z_{k}\right)= \\
& =\sum_{\mathrm{P}_{1}: Y=\bigcup_{i} X_{i}}(-1)^{\left|\mathrm{P}_{1}\right|-1}\left(\left|\mathrm{P}_{1}\right|-1\right) ! \sum_{X_{j} \subset P_{1}}\left(-\mathcal{L}_{\left|X_{j}\right|}\left(X_{j}\right)\right) \prod_{X_{i} \subset \mathrm{P}_{1}} S_{\left|X_{i}\right|}\left(-t, X_{i}\right) \times \\
& \quad \times \sum_{\mathrm{P}_{2}: X_{i}=\bigcup_{k} Z_{k}} \prod_{Z_{k} \subset \mathrm{P}_{2}} g_{\left|Z_{k}\right|}\left(0, Z_{k}\right) .
\end{aligned}
$$

In view of the equality

$$
S_{\left|X_{i}\right|}\left(-t, X_{i}\right) \sum_{P_{2}: X_{i}=\bigcup_{k} Z_{k}} \prod_{Z_{k} \subset \mathrm{P}_{2}} g_{\left|Z_{k}\right|}\left(0, Z_{k}\right)=\sum_{\mathrm{P}_{2}: X_{i}=\bigcup_{i} Z_{k}} \prod_{Z_{k} \subset \mathrm{P}_{2}} g_{\left|Z_{k}\right|}\left(t, Z_{k}\right),
$$


which follows from (9), we have

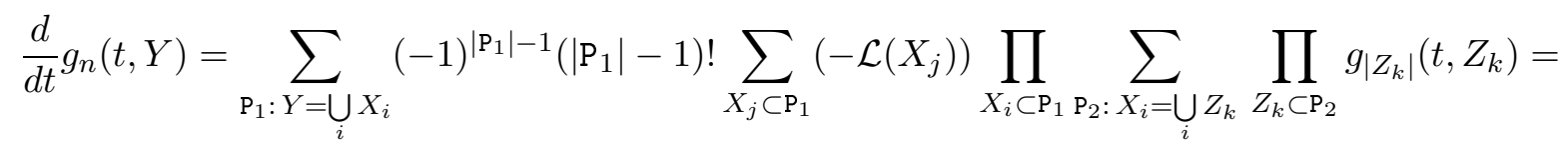

$$
\begin{aligned}
& =\left(-\mathcal{L}_{|Y|}(Y)\right) g_{n}(Y)+\sum_{\substack{\mathrm{P}_{1}: Y=\bigcup_{i} X_{i} \mathrm{P}_{2}: \mathrm{P}_{X_{i}}=\bigcup_{k} Z_{k} \\
\mathrm{P}_{1}>1}}(-1)^{\left|\mathrm{P}_{2}\right|-1}\left(\left|\mathrm{P}_{2}\right|-1\right) ! \times \\
& \times \sum_{Z_{k} \subset \mathrm{P}_{2}}\left(-\mathcal{L}_{\left|Z_{k}\right|}\left(Z_{k}\right)\right) \prod_{X_{i} \subset \mathrm{P}_{1}} g_{\left|X_{i}\right|}\left(t, X_{i}\right),
\end{aligned}
$$

the notation being similar to that in (44) and $Y_{X_{i}} \equiv\left(X_{1}, \ldots, X_{|\mathrm{P}|}\right)$ is the set whose elements are $|\mathrm{P}|$ subsets $X_{i} \subset Y$.

Since the following identity holds

$$
\sum_{\mathrm{P}: Y_{X_{i}}=\bigcup_{k} Z_{k}}(-1)^{|\mathrm{P}|-1}(|\mathrm{P}|-1) ! \sum_{Z_{k} \subset \mathrm{P}}\left(-\mathcal{L}_{\left|Z_{k}\right|}\left(Z_{k}\right)\right)=\sum_{Z_{i} \subset X_{i}}\left(-\mathcal{L}_{\left|\bigcup_{i=1}^{\text {int }} Z_{i}\right|}\left(\bigcup_{i=1}^{|\mathrm{P}|} Z_{i}\right)\right),
$$

where $\sum_{Z_{i} \subset X_{i}}$ is a sum over all nonempty subsets $Z_{i} \subset X_{i}$ and operator $\mathcal{L}_{\mid \bigcup_{i=1}^{i n t}}^{\text {in }} Z_{i} \mid$ is defined by formulas (3), we come to equations (11). Thus, formula (4) determines the solution of the initialvalue problem of the nonlinear Liouville hierarchy (11) from the viewpoint of the point-by-point convergence.

Let us show that the strong derivative of the solution (44), reproduces the generator of the nonlinear Liouville hierarchy (10) in the subspace $L_{n, 0}^{1} \subset L_{n}^{1}$.

For $g_{n}(0) \in L_{n, 0}^{1}$, in the sense of convergence norm of the space $L_{n}^{1}$, we have

$$
\begin{aligned}
& \lim _{\Delta t \rightarrow 0} \| \sum_{\mathrm{P}: Y=\bigcup_{i} X_{i} \mathrm{P}^{\prime}: Y_{X_{i}}=\bigcup \begin{array}{l}
Z_{k} \\
Z_{k}
\end{array}}(-1)^{\left|\mathrm{P}^{\prime}\right|-1}\left(\left|\mathrm{P}^{\prime}\right|-1\right) !\left[\frac{1}{\Delta t} \times\right. \\
& \times\left[\prod_{Z_{k} \subset \mathrm{P}^{\prime}} S_{\left|Z_{k}\right|}\left(-(t+\Delta t), Z_{k}\right) \prod_{X_{i} \subset \mathrm{P}} g_{\left|X_{i}\right|}\left(0, X_{i}\right)-\prod_{Z_{k} \subset \mathrm{P}^{\prime}} S_{\left|Z_{k}\right|}\left(-t, Z_{k}\right) \prod_{X_{i} \subset \mathrm{P}} g_{\left|X_{i}\right|}\left(0, X_{i}\right)\right]- \\
& \left.-\sum_{Z_{i} \subset P^{\prime}}\left(-\mathcal{L}_{\left|Z_{i}\right|}\left(Z_{i}\right)\right) \prod_{Z_{i} \subset \mathrm{P}^{\prime}} S_{\left|Z_{i}\right|}\left(-t, Z_{i}\right) \prod_{X_{i} \subset \mathrm{P}} g_{\left|X_{i}\right|}\left(0, X_{i}\right)\right] \|=0 .
\end{aligned}
$$

Indeed, within the context of the corollary of the Liouville theorem and the group property of operators $S_{n}(-t), n \geq 1$, (6) we obtain

$$
\begin{aligned}
& \lim _{\Delta t \rightarrow 0} \| \sum_{\mathrm{P}: Y=\bigcup_{i} X_{i} \mathrm{P}^{\prime}: Y_{X_{i}}=\bigcup_{k} Z_{k}}(-1)^{\left|\mathrm{P}^{\prime}\right|-1}\left(\left|\mathrm{P}^{\prime}\right|-1\right) !\left[\frac{1}{\Delta t} \times\right. \\
& \times\left[\prod_{Z_{k} \subset \mathrm{P}^{\prime}} S_{\left|Z_{k}\right|}\left(-(t+\Delta t), Z_{k}\right) \prod_{X_{i} \subset \mathrm{P}} g_{\left|X_{i}\right|}\left(0, X_{i}\right)-\prod_{Z_{k} \subset \mathrm{P}^{\prime}} S_{\left|Z_{k}\right|}\left(-t, Z_{k}\right) \prod_{X_{i} \subset \mathrm{P}} g_{\left|X_{i}\right|}\left(0, X_{i}\right)\right]- \\
& \left.-\sum_{Z_{i} \subset P^{\prime}}\left(-\mathcal{L}_{\left|Z_{i}\right|}\left(Z_{i}\right)\right) \prod_{Z_{i} \subset \mathrm{P}^{\prime}} S_{\left|Z_{i}\right|}\left(-t, Z_{i}\right) \prod_{X_{i} \subset \mathrm{P}} g_{\left|X_{i}\right|}\left(0, X_{i}\right)\right] \|= \\
& =\lim _{\Delta t \rightarrow 0} \| \sum_{\mathrm{P}: Y=\bigcup_{i} X_{i} \mathrm{P}^{\prime}: Y_{X_{i}}=\bigcup \begin{array}{l}
Z_{k} \\
Z_{k}
\end{array}}(-1)^{\left|\mathrm{P}^{\prime}\right|-1}\left(\left|\mathrm{P}^{\prime}\right|-1\right) !\left[\frac { 1 } { \Delta t } \left[\prod_{Z_{k} \subset \mathrm{P}^{\prime}} S_{\left|Z_{k}\right|}\left(-\Delta t, Z_{k}\right) \times\right.\right. \\
& \left.\left.\times \prod_{X_{i} \subset \mathrm{P}} g_{\left|X_{i}\right|}\left(0, X_{i}\right)-\prod_{X_{i} \subset \mathrm{P}} g_{\left|X_{i}\right|}\left(0, X_{i}\right)\right]-\sum_{Z_{i} \subset P^{\prime}}\left(-\mathcal{L}_{\left|Z_{i}\right|}\left(Z_{i}\right)\right) \prod_{X_{i} \subset \mathrm{P}} g_{\left|X_{i}\right|}\left(0, X_{i}\right)\right] \|=
\end{aligned}
$$




$$
\begin{aligned}
& =\int d Y \mid \sum_{\mathrm{P}: Y=\bigcup_{i} X_{i} \mathrm{P}^{\prime}: Y_{X_{i}}=\bigcup \begin{array}{l}
k \\
Z_{k}
\end{array}}(-1)^{\mid \mathrm{P}^{\prime}-1}\left(\left|\mathrm{P}^{\prime}\right|-1\right) ! \lim _{\Delta t \rightarrow 0}\left[\frac { 1 } { \Delta t } \left[\prod_{Z_{k} \subset \mathrm{P}^{\prime}} S_{\left|Z_{k}\right|}\left(-\Delta t, Z_{k}\right) \times\right.\right. \\
& \left.\left.\times \prod_{X_{i} \subset \mathrm{P}} g_{\left|X_{i}\right|}\left(0, X_{i}\right)-\prod_{X_{i} \subset \mathrm{P}} g_{\left|X_{i}\right|}\left(0, X_{i}\right)\right]-\sum_{Z_{i} \subset P^{\prime}}\left(-\mathcal{L}_{\left|Z_{i}\right|}\left(Z_{i}\right)\right) \prod_{X_{i} \subset \mathrm{P}} g_{\left|X_{i}\right|}\left(0, X_{i}\right)\right] .
\end{aligned}
$$

The last equality is valid becouse the integrand in this expression tends to zero as $\Delta t \rightarrow 0$ uniformly in $Y$ on any compact set. Therefore, we can pass to the limit as $\Delta t \rightarrow 0$ in the integral. Hence, equality (4) is differentiable in the norm of the space $L_{n}^{1}$. Thus, for the initial data from $L_{n, 0}^{1} \subset L_{n}^{1}$ the corresponding Cauchy problem has a unique strong solution that given by expansions (1)-(2).

\section{Properties of the solution of the nonlinear Liouville hierarchy.}

We introduce the evolution operator of the solution (4)

$$
\sum_{\mathrm{P}: Y=\bigcup_{i} X_{i}} \mathfrak{A}_{|\mathrm{P}|}\left(t, Y_{X_{i}}\right) \prod_{X_{i} \subset \mathrm{P}} g_{\left|X_{i}\right|}\left(0, X_{i}\right) \equiv\left(\mathfrak{A}_{t}(g(0))\right)_{|Y|}(Y) .
$$

For the evolution operator (10), the group property is valid, i.e.,

$$
\left(\mathfrak{A}_{t_{1}}\left(\mathfrak{A}_{t_{2}}(g(0))\right)\right)_{|Y|}(Y)=\left(\mathfrak{A}_{t_{2}}\left(\mathfrak{A}_{t_{1}}(g(0))\right)\right)_{|Y|}(Y)=\left(\mathfrak{A}_{t_{1}+t_{2}}(g(0))\right)_{|Y|}(Y) .
$$

Indeed, for $g_{n}(0) \in L_{n}^{1}, n \geq 1$ and for any $t_{1}, t_{2} \in \mathbb{R}^{1}$, according to the notation (4) and (5)), we have

$$
\begin{aligned}
\left(\mathfrak{A}_{t_{1}}\right. & \left.\left(\mathfrak{A}_{t_{2}}(g(0))\right)\right)_{|Y|}(Y)= \\
& =\sum_{\mathrm{P}: Y=\bigcup_{i} X_{i}} \mathfrak{A}_{|\mathrm{P}|}\left(t_{1}, Y_{X_{i}}\right) \prod_{X_{i} \subset \mathrm{P} \mathrm{P}^{\prime}: X_{i}=\bigcup \begin{array}{l}
l \\
Z_{l}
\end{array}} \mathfrak{A}_{\left|\mathrm{P}^{\prime}\right|}\left(t_{2},\left(X_{i}\right)_{Z_{l}}\right) \prod_{Z_{i} \subset \mathrm{P}^{\prime}} g_{\left|Z_{l}\right|}\left(0, Z_{l}\right)= \\
& =\sum_{\mathrm{P}: Y=\bigcup_{i} X_{i} \mathrm{P}_{1}: Y_{X_{i}}=\bigcup_{k} Q_{k}}(-1)^{\left|\mathrm{P}_{1}\right|-1}\left(\left|\mathrm{P}_{1}\right|-1\right) ! \prod_{Q_{k} \subset \mathrm{P}^{\prime}} S_{\left|Q_{k}\right|}\left(-t_{1}, Q_{k}\right) \prod_{X_{i} \subset \mathrm{P}} \times \\
& \times \sum_{\mathrm{P}^{\prime}: X_{i}=\bigcup_{l} Z_{l} \mathrm{P}_{2}:\left(X_{i}\right)_{Z_{l}}=\bigcup_{j} R_{j}}(-1)^{\left|\mathrm{P}_{2}\right|-1}\left(\left|\mathrm{P}_{2}\right|-1\right) ! \prod_{R_{k} \subset \mathrm{P}_{2}} S_{\left|R_{k}\right|}\left(-t_{2}, R_{k}\right) \prod_{Z_{l} \subset \mathrm{P}^{\prime}} g_{\left|Z_{l}\right|}\left(0, Z_{l}\right) .
\end{aligned}
$$

Having collected the items at identical products of the initial data $g_{n}(0), n \geq 1$, and taking into account the group property of the evolution operators $S_{n}(-t), n \geq 1$ (6) , we obtain

$$
\begin{aligned}
\left(\mathfrak{A}_{t_{1}}\right. & \left.\left(\mathfrak{A}_{t_{2}}(g(0))\right)\right)_{|Y|}(Y)= \\
& =\sum_{\mathrm{P}: Y=\bigcup_{i}} \sum_{X_{i} \mathrm{P}^{\prime}: Y_{X_{i}}=\bigcup_{l} Z_{l}}(-1)^{\left|\mathrm{P}^{\prime}\right|-1}\left(\left|\mathrm{P}^{\prime}\right|-1\right) ! \prod_{Z_{l} \subset \mathrm{P}^{\prime}} S_{\left|Z_{l}\right|}\left(-\left(t_{1}+t_{2}\right), Z_{l}\right) \prod_{X_{i} \subset \mathrm{P}} g_{\left|X_{i}\right|}\left(0, X_{i}\right)= \\
& =\sum_{\mathrm{P}: Y=\bigcup_{i} X_{i}} \mathfrak{A}_{|\mathrm{P}|}\left(t_{1}+t_{2}, Y_{X_{i}}\right) \prod_{X_{i} \subset \mathrm{P}} g_{\left|X_{i}\right|}\left(0, X_{i}\right)=\left(\mathfrak{A}_{t_{1}+t_{2}}(g(0))\right)_{n}(Y) .
\end{aligned}
$$

Similarly,

$$
\left(\mathfrak{A}_{t_{2}}\left(\mathfrak{A}_{t_{1}}(g(0))\right)\right)_{n}(Y)=\left(\mathfrak{A}_{t_{1}+t_{2}}(g(0))\right)_{n}(Y) .
$$


Let us consider the property of the solution (4) for one physically motivated example of the initial data that is to say if the initial data for Cauchy problem (11)-(2), satisfy the 'chaos' condition 4, in other words, the sequences of correlation functions have the form

$$
g(0)=\left(1, g_{1}\left(0, x_{1}\right), 0,0, \ldots\right)
$$

Indeed, in terms of the sequences $D(0)$, this condition implies [4] that

$$
D(0)=\left(1, D_{1}\left(0, x_{1}\right), D_{1}\left(0, x_{1}\right) D_{1}\left(0, x_{2}\right), \ldots\right),
$$

which implies that particle distributions are statistically independent at the initial time instant. Making use of the relation (8), we obtain the initial condition (11) for the correlation functions.

For the initial data (11), the formula for the solution (4) of the initial-value problem (11)-(2) is simplified and reduces to

$$
g_{n}\left(t, x_{1}, \ldots, x_{n}\right)=\mathfrak{A}_{n}\left(t, x_{1}, \ldots, x_{n}\right) \prod_{i=1}^{n} g_{1}\left(0, x_{i}\right) .
$$

In this case the following estimate holds

$$
\left\|g_{n}(t)\right\| \leq n ! e^{n+1}\left\|g_{1}(0)\right\|^{n} .
$$

In the case of the initial data (11), the solution (12) of the Cauchy problem for the nonlinear Liouville hierarchy may be rewritten in a different form. If $n=1$, we have

$$
g_{1}\left(t, x_{1}\right)=\mathfrak{A}_{1}\left(t, x_{1}\right) g_{1}\left(0, x_{1}\right)=g_{1}\left(0, q_{1}-p_{1} t, p_{1}\right)
$$

i.e., an explicit expression that describes the evolution of one particle. Then, within the context of the definition of the 1 -st order cumulant, $\mathfrak{A}_{1}(t)$, and inverse to it evolution operator $\mathfrak{A}_{1}(-t)$, we can express the correlation functions $g_{n}(t), n \geq 2$, in terms of the one-particle correlation function $g_{1}(t)$ making use of formula (12).

Finally, formula (12) for $n \geq 2$ is given by

$$
g_{n}\left(t, x_{1}, \ldots, x_{n}\right)=\widehat{\mathfrak{A}}_{n}\left(t, x_{1}, \ldots, x_{n}\right) \prod_{i=1}^{n} g_{1}\left(t, x_{i}\right),
$$

where $\widehat{\mathfrak{A}}_{n}\left(t, x_{1}, \ldots, x_{n}\right)$ is the $n$-th order cumulant of the scattering operators $\widehat{S}_{t}$, i.e.,

$$
\widehat{S}_{t}\left(x_{1}, \ldots, x_{n}\right)=S_{n}\left(-t, x_{1}, \ldots, x_{n}\right) \prod_{i=1}^{n} S_{1}\left(t, x_{i}\right)
$$

whose generators are determined by the operator $\mathcal{L}^{\text {int }}(\underline{3})$ in terms of the interaction potential of the system.

\section{Acknowledgement}

The author is pleased to thank Prof. Victor Gerasimenko for the formulation of the problem and for many useful discussions. 
[1] Bogolyubov N. On the stochastic processes in dynamical systems.- in EPAN, Dubna: JINR, 9, N 4, 1978.

[2] Green M.S. Boltzmann equation from the statistical mechanical point of view.- Journal Chem. Phys., 25, N 5, 1956, pp. 836-855.

[3] Gerasimenko V.I. and Ryabukha T.V., Cumulant representation of solutions of the BBGKY hierarchy of equations, Ukr. Math. J.. - $2002-\mathbf{5 4}$, N 10. - pp. 1583-1601.

[4] Cercignani C., Gerasimenko V.I., Petrina D.Ya. Many-Particle Dynamics and Kinetic Equations. - Kluwer Acad. Publ., 1997. - p. 256.

[5] Gerasimenko V. I., Ryabukha T. V., Stashenko M. O. On the structure of expansions for the BBGKY Hierarchy Solutions // J. Phys. A: Math. Gen. - 2004. - 37. - pp. 9861-9872.

[6] Petrina D.Ya., Gerasimenko V.I., Malyshev P.V. Mathematical Foundations of Classical Statistical Mechanics. Continuous Systems. - London and N.Y.: Taylor \& Francis Inc. (Second ed.), 2002. - p. 352.

[7] Bogolyubov N. Lectures on Quantum Statistics.- Kyiv: Rad. shkola, 1949. 